\title{
A trim problem formulation for maximum control authority using the Attainable Moment Set geometry
}

\author{
Carmine Varriale $^{1}$ (D) $\cdot$ Mark Voskuijl ${ }^{2}$
}

Received: 18 May 2021 / Revised: 7 September 2021 / Accepted: 13 October 2021 / Published online: 26 November 2021

(c) The Author(s) 2021

\begin{abstract}
This paper presents a generic trim problem formulation, in the form of a constrained optimization problem, which employs forces and moments due to the aircraft control surfaces as decision variables. The geometry of the Attainable Moment Set (AMS), i.e. the set of all control forces and moments attainable by the control surfaces, is used to define linear equality and inequality constraints for the control forces decision variables. Trim control forces and moments are mapped to control surface deflections at every solver iteration through a linear programming formulation of the direct Control Allocation algorithm. The methodology is applied to an innovative box-wing aircraft configuration with redundant control surfaces, which can partially decouple lift and pitch control, and allow direct lift control. Novel trim applications are presented to maximize control authority about the lift and pitch axes, and a "balanced" control authority. The latter can be intended as equivalent to the classic concept of minimum control effort. Control authority is defined on the basis of control forces and moments, and interpreted geometrically as a distance within the AMS. Results show that the method is able to capitalize on the angle of attack or the throttle setting to obtain the control surfaces deflections which maximize control authority in the assigned direction. More conventional trim applications for minimum total drag and for assigned angle of elevation are also explored.
\end{abstract}

Keywords Flight mechanics · Flight control · Control Allocation · Trim · Attainable Moment Set · Box-wing aircraft

\section{List of symbols}

$\mathcal{A} \quad$ Control authority

$B \quad$ Control effectiveness matrix, $1 / \mathrm{rad}$

$C_{(} \quad$ Generic non-dimensional coefficient

$D \quad$ Drag force, $\mathrm{N}$

$\boldsymbol{F} \quad$ Generalized forces, $\mathrm{N}$ or $\mathrm{N}-\mathrm{m}$

$\mathcal{J} \quad$ Generic objective function

$L \quad$ Lift force, $\mathrm{N}$

$M_{\infty} \quad$ Asymptotic Mach number

$\mathcal{L}, \mathcal{M}, \mathcal{N} \quad$ Roll, pitch, yaw moments, $\mathrm{N}-\mathrm{m}$

$V \quad$ Airspeed, $\mathrm{m} / \mathrm{s}$

$X_{E}, Y_{E}, Z_{E}$ Position in Earth reference frame, $\mathrm{m}$

$f \quad$ Equations of motion

$f_{\mathrm{CA}} \quad$ Control Allocation method

$\ell \quad$ Half-line in Moment Space

$p, q, r$
$\boldsymbol{u}, \boldsymbol{x}$
$\alpha, \beta, \gamma$
$\phi, \theta, \psi$
$\boldsymbol{\delta}$
$\delta_{T}$
$\boldsymbol{\tau}$

Angular rates in body axes, $\mathrm{rad} / \mathrm{s}$

Dynamic system inputs and states

Angles of attack, side-slip, flight path, rad

Angles of bank, elevation, heading, rad

Control effectors positions, rad

Throttle level

Trim controls

Subscripts and superscripts

$\mathrm{CH} \quad$ Convex hull

GT Ground track

SW Side wind

eq, ineq Equality, inequality constraint

$\mathrm{lb}$, ub Lower bound, upper bound

ref Reference condition

tr Trim condition
Carmine Varriale

C.Varriale@tudelft.nl

1 Flight Performance and Propulsion Section, Faculty of Aerospace Engineering, Delft University of Technology, 2629 HS Delft, The Netherlands

2 Faculty of Military Sciences, Netherlands Defence Academy, 1780 AC Den Helder, The Netherlands

\section{Introduction}

Trimming a dynamic system means finding the combination of input and state variables values which set the system in a steady-state condition [1]. This paper presents a novel generic trim problem formulation, in the form of a 
constrained optimization problem, which employs forces and moments due to the aircraft control surfaces as decision variables. The constraints (and, optionally, the objective function) of such optimization problem are obtained by exploiting the geometric properties of the Attainable Moment Set (AMS). The latter is a convex polytope representing all the possible forces and moments that can be attained by the aircraft control effectors and flight control system [2]. Control forces and moments are then mapped to the effectors using a linear programming (LP) formulation of the Direct Control Allocation (DCA) method [3].

Using control forces and moments as decision variables, the proposed trim formulation is well suited to be applied to aircraft configurations with a high number of control surfaces. By relying on the geometry of the AMS, the formulation makes it possible to objectively define the feasible region of the trim optimization problem, exclusively on the basis of the aircraft control effectiveness. By relying on a CA method to link control forces and moments to control surface deflections, the formulation also ensures that trim solutions can automatically be obtained by an appropriate, practical realization of the aircraft flight control system.

The paper formalizes, streamlines and expands the previous research effort, reported in [4]. With respect to the previous work, several new applications are presented for a box-wing aircraft configuration.

First, the possibility to trim for maximum control authority in a specific direction of one or more motion axes is explored. In this case, the AMS geometry is not only used to generate linear constraints for the trim problem, but also used to calculate the objective function itself. New examples in symmetric flight are provided, with an analysis of the effect of airspeed on control authority and other flight parameters. A 6 Degrees of Freedom (DoF) application to asymmetric flight in side wind conditions is reported for completeness. The latter is interesting to observe the behavior of control surface deflections in complex flight scenarios.

Control authority is defined as the ability of the control effectors to generate forces and moments in the given direction of the selected set of motion axes. Examples may include control authority to pitch up, or to lift up and down (as in the case of spoilers). The concept of a maximum "balanced" control authority, about all motion axes, would be equivalent to the one of minimum total control effort. Searching for trim conditions which guarantee rotational equilibrium and at the same time increase the control authority for pitch-up maneuvers could be interesting for safety purposes, for example in case of aborted landings.

A similar scenario can be envisioned to justify the interest in trim with maximum lift-up control authority. Because lift lies perpendicular to the velocity vector, by definition, an increase in lift introduces a centripetal acceleration $V \dot{\gamma}$ which is the most direct way to bend the trajectory upwards. The study of control authority in the lift axis is particularly aimed at exploiting one of the most interesting capabilities of the box-wing aircraft configuration: the innovative way of implementing direct lift control (DLC) [5, 6].

More traditional applications studies have also been performed, focusing on trim conditions for minimum drag and for a specified angle of elevation. In the first study, the impact of the set of allocated control forces on the objective function is monitored. In the second study, the ability of box-wing aircraft to (partially) decouple vertical and rotational equilibrium is tested.

The following Sect. 2 presents and reviews several trim problem formulations proposed in the available literature. The flight mechanics and simulation model is then briefly presented in Sect. 3. Section 4 defines and formalizes the concept of control authority in the scope of CA theory, while the new trim problem formulation is illustrated in Sect. 5. Results are shown in Sect. 6 for all the applications on control authority, minimum drag and assigned elevation angle. Lastly, conclusions are drawn in Sect. 7, with suggestions on future research.

\section{Literature review}

In the most general case, system dynamics is expressed by non-linear, implicit or explicit equations, as shown in the following Eq. (1).

$f(\dot{x}, x, u)=0$

If the system is trimmed, none of the states is changing in time and Eq. (2) holds. The trim problem consists in finding the values of $\boldsymbol{x}^{\text {tr }}$ and $\boldsymbol{u}^{\text {tr }}$ such that Eq. (2) is verified.

$\boldsymbol{f}\left(\dot{\boldsymbol{x}}^{\mathrm{tr}}=\mathbf{0}, \boldsymbol{x}^{\mathrm{tr}}, \boldsymbol{u}^{\mathrm{tr}}\right)=\mathbf{0}$

In general, if $\mathbb{S}=\{\boldsymbol{x}, \boldsymbol{u}\}$ is the set of system states and inputs, some subset $\tau_{0} \subseteq \mathbb{S}$ can (or must) be characterized explicitly in order to define the desired trim condition. The variables belonging to this subset have, therefore, known values. The remaining subset $\boldsymbol{\tau}=\mathbb{S}-\boldsymbol{\tau}_{0}$ contains unknown variables, which are referred to as "trim controls". Using these definitions, it is possible to represent $\mathbb{S}^{\text {tr }}$ either in terms of system states and inputs, or in terms of assigned and unknown variables, as done in Eq. (3). The trim problem consists then in determining the values of the trim controls $\tau$ such that Eq. (2) is verified.

$\mathbb{S}^{\text {tr }}=\left\{\boldsymbol{x}^{\mathrm{tr}}, \boldsymbol{u}^{\mathrm{tr}}\right\}=\left\{\boldsymbol{\tau}_{0}, \boldsymbol{\tau}\right\}$

Formulations of the trim problem can be classified on the basis of the number of dynamic equations $N_{f}$ and the number of trim controls $N_{\tau}$. The trim problem is said to be over-determined if $N_{f}>N_{\tau}$, determined if $N_{f}=N_{\tau}$, or under-determined if $N_{f}<N_{\tau}$. This classification does not 
give any indication on the number of solutions that the trim problem can have. Due to non-linearities and couplings in the dynamic equations, even a determined trim problem can have zero or more than one solution. But the complexity and the number of possible solutions of a given trim problem usually increases with the number of trim controls, making under-determined problems generally tougher to solve than determined ones.

An extensive and detailed analysis of the classic trim problem for rigid aircraft dynamics with $6 \mathrm{DoF}$ is presented in [7]. The trim problem is formulated as in Eq. (4) and assumes the aircraft is trimmed when the objective function approaches zero within a certain tolerance. It is therefore, in essence, a root-finding problem.

$$
\min _{\tau} \mathcal{J}=\|\dot{x}\|^{2}
$$

subj. to $\tau_{\mathrm{lb}} \leq \boldsymbol{\tau} \leq \tau_{\mathrm{ub}}$

For every flight condition, the number of assigned trim parameters $\tau_{0}$ is chosen so that the trim problem is determined. By limiting the scope to $6 \mathrm{DoF}$ dynamic models of conventional aircraft configurations, each trim problem can be designed to have exactly six trim controls: the four conventional control effectors (throttle, elevator, aileron pair, and rudder), and two Euler angles. This solid approach may show its limitations when considering aircraft configurations with higher number of control inputs, e.g. with redundant or unganged sets of control effectors, for which the trim problem becomes under-determined.

An early attempt at solving an under-determined trim problem for aircraft longitudinal dynamics is provided in [8]. The trim problem is formulated as an induced drag minimization problem, with constraints on the vertical and rotational equilibrium in the longitudinal axis. The equations of motion are linearized with classic assumptions for the cruise condition and a closed form solution is derived. Examples are provided for an aircraft with three-lifting surfaces and a fighter jet with thrust vectoring capabilities. In both cases, $N_{\tau}=N_{f}+1$ and it is possible to minimize a single scalar parameter, induced drag, while trimming the aircraft.
The case of under-determined trim problems due to control effectors redundancy has been analyzed in [9], with applications to the Blended Wing Body (BWB) aircraft configuration. Two trim problem formulations are proposed:

1. A Minimum Drag Trim Optimization (MDTO) applied to 6 DoF dynamics.

2. A Root-Finding Trim with Direct Allocation (RFTDA), analogous in the objective function to the one in Eq. (4).

For the MDTO formulation, unganged control surface deflections are directly included in the the trim controls set, i.e. $\delta \in \tau$. In this way, the dimension of $\tau$ increases linearly with the amount of control effectors, making the trim problem more complex for highly redundant aircraft configurations. For the RFTDA formulation, the trim controls set does not contain the control surface deflections, but a set of aerodynamic actions due to the control effectors $\Delta \boldsymbol{F}$. With this approach, the dimension of $\boldsymbol{\tau}$ does not depend on the control surface redundancy of the aircraft configuration. A DCA method, is used to establish a relation between $\Delta \boldsymbol{F}$ and $\boldsymbol{\delta}$. Lower and upper bounds for the $\Delta \boldsymbol{F}$ trim controls are selected on the basis of previously obtained solutions, or by manually inspecting particular combinations of control surface deflections for the specific problem.

A synthetic recap of the reviewed trim problem formulations is shown in Table 1. Comparing the two approaches presented in [9] would be overall unfair. The MDTO is an optimization-based approach that exploits controls redundancy to minimize drag at trim conditions. The RFTDA is a root-finding trim approach that copes with control redundancy through a CA algorithm. No parameter is explicitly optimized with the latter formulation and the method is indeed incapable of returning the minimum drag trim condition.

With reference to the RFTDA approach, the dimension of $\Delta \boldsymbol{F}$, here indicated with $N_{\boldsymbol{F}}$, is somewhat arbitrary and depends on which motion axes are selected to be controlled. $\Delta \boldsymbol{F}$ contains up to two elements (lift and pitch moment) for flight simulations constrained in the longitudinal plane, or up to four elements (lift, and roll, pitch, yaw moments) for 6 DoF simulations. It is noted that the number and type of
Table 1 Summary of trim problem formulations from the reviewed literature

\begin{tabular}{lllll}
\hline Ref. & DoF & Secondary objective & Trim controls & Control Allocation \\
\hline$[7]$ & 6 & None & $\theta, \psi, \delta, \delta_{T}$ & Ganging \\
{$[8]$} & 3 & Drag & $C_{L_{1}}, C_{L_{2}}, C_{L_{3}}$ & Not modeled \\
& 3 & Drag & $C_{L_{1}}, C_{L_{2}}, \delta_{T}$ & Not modeled \\
{$[9]$} & 3,6 & None, drag & $\alpha, \theta, \delta, \delta_{T}$ & None \\
& 3 & None & $\alpha, \theta, \mathcal{M}, \delta_{T}$ & DCA \\
& 3 & None & $\alpha, \theta, \mathcal{M}, L, \delta_{T}$ & DCA \\
& 6 & None & $\alpha, \theta, \psi, \phi, \mathcal{M}, \mathcal{N}, L, \delta_{T}$ DCA \\
\hline
\end{tabular}


elements in $\Delta \boldsymbol{F}$ has a significant impact on the resulting control surface deflections at trim. In particular, introducing lift in the trim control vector (hence, also in the DCA problem) results in trim conditions with better aerodynamic efficiency, i.e. lower drag. Control drag is not explicitly included in $\tau$, because the DCA would poorly approximate its quadratic behavior with deflection angles.

Despite traditional CA literature considers only the allocation of the roll, pitch and yaw control moments (the socalled "three moment problem" [2]), several research efforts have been proposed to allocate control forces as well. These are mostly targeted to aircraft configurations with redundant control effectors, which "have the capability to allocate commands for a larger number of objectives" and for which "direct force commands can be applied in addition to moment commands [10]". A fundamental effort is provided by [10], where the geometry-based algorithm conceived to solve the three-moment DCA problem is generalized to be applicable to four and more allocation objectives. In such work, the AMS is addressed in a more abstract way as the "Attainable Objective Set". As long as all of the allocated forces and moments are in the form of dimensionless coefficients, the geometry of the CA problem scales coherently with the dimension of the vector of objectives, and no additional considerations must be made on the physical nature of the objectives themselves [10].

Recent applications to direct force allocation include the already mentioned RFTDA trim approach [9], which incorporates lift in the trim decision variables and allocates it to control surfaces with a DCA algorithm. Additionally, advanced CA algorithms that explicitly minimize control drag have been developed in the past, but have never been applied to formulations of the trim problem. An incremental, or frame-wise, expansion of the DCA method is presented in [11]. A model-specific incremental CA method is presented in [12], where drag is expressed as a quadratic function of the effectors, and the CA algorithm solves a quadratic programming optimization problem.

After a brief overview of the flight mechanics model used to perform all the trim simulations presented in the remainder of this paper, relevant aspects of the CA problem are introduced in more detail in Sect. 4.

\section{Flight mechanics model}

The concepts developed in this work apply to any aircraft configuration, with any level of control redundancy. The aircraft model used as the main application case for this paper is an unconventional, box-wing configuration referred to as the PrandtlPlane (PrP). The specific PrP used for this study is a 300 passenger, transonic, commercial transport aircraft, which has the been the object of the PARSIFAL research

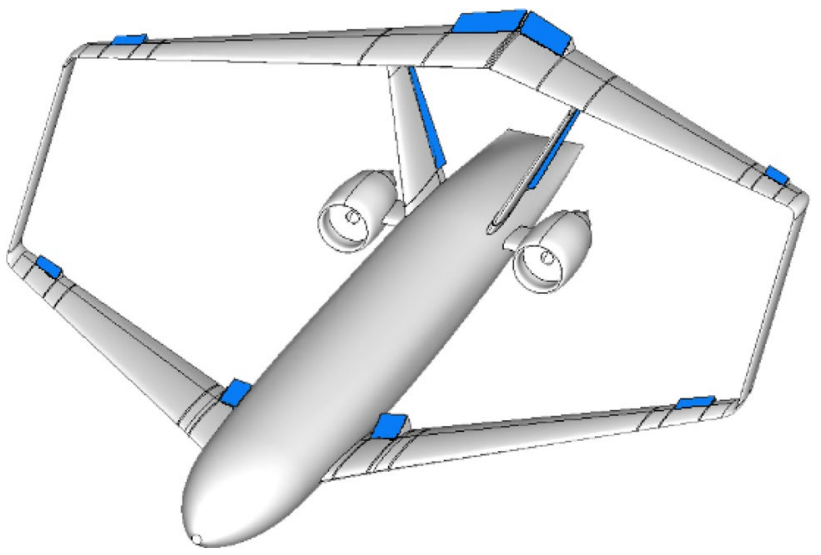

Fig. 1 PrP aircraft configuration, with highlighted control surfaces

project $[13,14]$, and is shown in Fig. 1. The box-wing has been known for a long time to be the "best wing system" for induced drag performance [15], and the PrP concept strives to integrate it in an innovative aircraft architecture for sustainable future aviation. The double wing system of the PrP allows the installation of multiple control effectors, which poses an interesting design challenge, and at the same time enables innovative control possibilities like DLC [29]. With control surfaces on the both the front and rear wings, the PrP is capable to generate substantial variations in lift, while decoupling, partially or totally, the control of pitch moment from the one of vertical forces.

The aircraft geometry is created in the Multi Model Generator, an in-house developed Knowledge-Based Engineering toolbox [16]. This tool provides automatic, configuration-agnostic modeling and meshing capabilities, which can be interfaced with selected aerodynamic solvers. By making use of the commercial panel method code VSAERO [17], a vast aerodynamic database for the PrP has been generated in the form of look-up tables. VSAERO calculates the potential flow around the assigned geometry, and corrects it in a series of iterations using integral boundary layer equations. Additionally, it can account for compressibility effects using the Karman-Tsien rule or the Prandtl-Glauert correction.

The database for steady aerodynamics expresses the six non-dimensional aerodynamic actions in Body Axes as tabular functions of $\alpha, \beta, M_{\infty}$ and control surface deflections $\delta$. Dynamic derivatives with respect to the angular rates $p, q, r$ are calculated for each flight condition with a second order finite difference formula. Each of the six aerodynamic actions is then expressed as in Eq. (5), assuming superposition of effects.

$$
\begin{gathered}
C_{F}=C_{F_{0}}\left(\alpha, \beta, M_{\infty}, \delta=\mathbf{0}\right)+\sum_{i=1}^{N_{\delta}} \Delta C_{F}\left(\alpha, \beta, M_{\infty}, \delta_{i}\right)+ \\
+\sum_{\omega=p, q, r} C_{F_{\omega}}\left(\alpha, \beta, M_{\infty}, \delta=\mathbf{0}\right) \omega
\end{gathered}
$$


For the aerodynamic database used in the present study, both $\alpha$ and $\beta$ range from $-6^{\circ}$ to $6^{\circ}$ in steps of $3^{\circ}$, while two Mach numbers have been analyzed, namely $M_{\infty}=0.3$ and $M_{\infty}=0.6$. Each control surface has been deflected, independently from all the others, from $-30^{\circ}$ to $30^{\circ}$ in steps of $10^{\circ}$.

The aerodynamic database is imported in the Performance, Handling Qualities, and Loads Analysis Toolbox (PHALANX). This is a non-linear simulation and analysis toolbox, which integrates data and sub-models from various aeronautical disciplines (aerodynamics, propulsion, flight control system, weight and balance, etc.), in order to generate a complete Flight Mechanics model of the aircraft. PHALANX is developed in MATLAB ${ }^{\circledR} / \mathrm{Sim}$ ulink and revolves around a Simscape Multibody Dynamics core to implement the equations of motion in aircraft body axes. The toolbox is aircraft configuration-agnostic and data-driven, meaning that its fidelity depends on the data and formulations used in the sub-models. This allows PHALANX to operate consistently at different stages of the aircraft design process.

The toolbox has been used in the past for the Flight Mechanics analysis of the PrP [5, 29], its mission performance evaluation [6], and the sizing of control surfaces on its box-wing geometry [18, 19]. PHALANX has also been employed in the analysis of different novel aircraft configurations like the BWB [20] and the Delft University Unconventional Configuration (DUUC), featuring the propulsive empennage concept [21].

\section{Background}

\subsection{The effective moment set}

The set of all forces and moments which can be generated by the control effectors is here referred to as the Effective Moment Set (EMS). The EMS is a function of the Admissible Controls Set (ACS), which is the set of all possible combinations of effectors positions. If the effectors positions are simply bounded, the ACS is a hyper-rectangle in Control Space, a Cartesian axis system in $\mathbb{R}^{N_{\delta}}$ with a control effector position varying on each axis.

Due to non-linearities and couplings in the aerodynamic model of control effectors, it is usually hard to characterize the EMS in Moment Space, a Cartesian axis system in $\mathbb{R}^{N_{F}}$ with a control force or moment varying on each axis. One notable analytic effort is presented in [22]. If the aerodynamic model of Eq. (5) is linearized with respect to the control effectors positions, the control effectiveness matrix defines a linear function which maps the ACS to an approximation of the EMS, as shown in Eq. (6).
$B=\frac{\partial C_{F}}{\partial \delta}=\frac{\partial \Delta C_{F}}{\partial \delta}: \Delta C_{F} \approx B \delta$

For a constant $B$ matrix and with the ACS being a convex set, it can be proven that the EMS is a bounded convex polytope in $\mathbb{R}^{N_{F}}$ [23], as shown in Fig. 2. The geometric algorithm to construct the EMS, given the $B$ matrix and the effectors positions saturation limits, is described in [2] for $N_{F}=2$ and $N_{F}=3$. For this work, it has been generalized to any number of Moment Space dimensions.

\subsection{Control Allocation and the Attainable Moment Set}

Every point $\Delta C_{F}$ in Moment Space can be associated with a combination of control effectors positions $\delta$. This task constitutes the fundamental CA problem and, in the scope of the linear formulation of Eq. (6), resolves in inverting the control effectiveness matrix $B$. If the matrix is square, i.e. $N_{F}=N_{\delta}$, the solution is unique and immediately determined. This is generally the case for conventional aircraft configurations with ganged control surfaces, where each effector is used to control one motion axis. Aircraft featuring redundant and/or unganged control effectors result in a $B$ matrix having more columns than

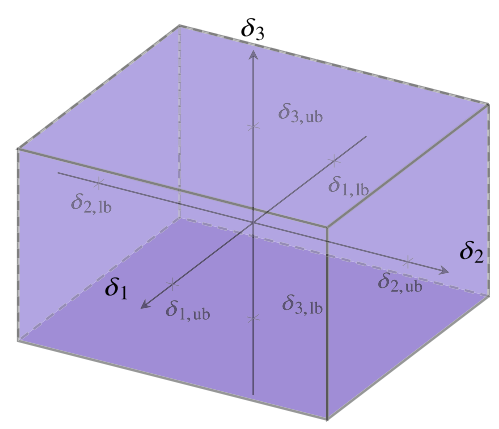

(a) Generic ACS in $\mathbb{R}^{3}$

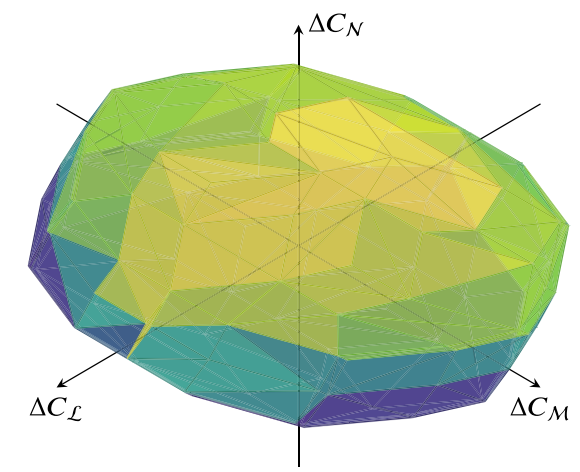

(b) Generic EMS in $\mathbb{R}^{3}$ for the classic "three moment" allocation problem

Fig. 2 Examples of a realistic Admissible Control Set (ACS) and Effective Moment Set (EMS) for a constant control effectiveness matrix $B$ 
rows, which, therefore, cannot be inverted exactly. A function or algorithm $f_{\mathrm{CA}}$ has then to be found to perform the $\mathrm{CA}$, as shown in the following Eq. (7).

$\delta=f_{\mathrm{CA}}\left(B, \Delta C_{\boldsymbol{F}}\right)$

A vast variety of CA methods has been developed in the past, for both linear and non-linear aerodynamic models. A detailed survey of CA methods is presented in [24], but most formulations entail the solution of an optimization problem. Some methods are targeted to simply minimize the difference between the desired and the attained control forces, as shown in Eq. (8), while others do so while optimizing a secondary parameter, like total effectors displacement, trim drag [11, 12], or structural loads [25].

$\min _{\delta} \mathcal{J}=\left\|\Delta C_{F}-B \delta\right\|$

As systematically demonstrated in [2], not all CA methods are capable of returning admissible control positions for every generalized force in the interior or on the boundary of the EMS. In other words, not all CA methods are capable of mapping the entirety of the EMS back to the ACS. The subset of the EMS which a CA method can trace to feasible positions of the effectors is here referred to as the AMS. The AMS is, in general, a subset of the EMS. Control forces and moments of the EMS which are outside of the AMS are unattainable by the given CA algorithm, despite being actually attainable by the control power available to the aircraft.

In the present work, the LP formulation of the DCA method presented in [3] is used. The LP-DCA method converts the classic, direction-preserving DCA problem reported in Eq. (9) to the smallest equivalent LP problem. In this relation, the scale factor $\rho$ is used to evaluate the control objectives in the same direction (in Moment Space) of the prescribed control forces and moments $\Delta C_{F}$, and to scale the resulting control surface deflections if $\Delta C_{F}$ are actually not attainable. For this algorithm, as for all DA methods, the AMS coincides with the EMS. Moreover, the LP formulation can be scaled to any number of controlled axes $N_{F}$, mixing forces and moments, without loss of computational efficiency.

$$
\begin{aligned}
\max _{\rho, \delta} & \mathcal{J}=\rho \\
\text { subj. to } & \rho \Delta C_{\boldsymbol{F}}-B \boldsymbol{\delta}=0 \\
& \boldsymbol{\delta}_{\mathrm{lb}} \leq \boldsymbol{\delta} \leq \boldsymbol{\delta}_{\mathrm{ub}}
\end{aligned}
$$$$
\text { with } \quad \delta \leftarrow \delta / \rho \text { if } \rho>1
$$

\subsection{Control authority}

As mentioned previously, control authority is here defined as the ability of the control effectors to generate forces and moments about one or more directions of Moment Space, from a given reference condition. It is here indicated with the symbol $\mathcal{A}_{F}$. For example, pitch-up and pitch-down control authorities can be respectively calculated as in Eqs. (10) and (11).

$\mathcal{A}_{+\mathcal{M}}=\left|C_{\mathcal{M}}^{\max }-C_{\mathcal{M}}^{\mathrm{ref}}\right|$

$\mathcal{A}_{-\mathcal{M}}=\left|C_{\mathcal{M}}^{\min }-C_{\mathcal{M}}^{\mathrm{ref}}\right|$

Although control authority is a function of all flight parameters, for a given flight condition $\left\{\alpha, \beta, M_{\infty}\right\}$ it only depends on the position of the control effectors. In this case, it can be expressed in terms of the aerodynamic actions due to the control effectors $\Delta C_{F}$, as shown in Eq. (12). It is, therefore, a quantity which lives in Moment Space, and in particular within the AMS.

$\Delta \mathcal{A}_{F}=\left\|\Delta C_{F}^{\lim }-\Delta C_{F}^{\mathrm{ref}}\right\|$

For a given control authority direction $\boldsymbol{F}$ in Moment Space, the limit point $\Delta C_{F}^{\lim }$ is found at the intersection of the AMS boundary and a half-line $\ell_{\boldsymbol{F}}$ starting at the reference point $\Delta C_{F}^{\text {ref }}$. Control authority finds its geometric representation as the distance between these two points of the AMS, as shown in Fig. 3.

\section{Methodology}

This section presents a comprehensive formulation of the trim problem for 6 DoF Flight Mechanics applications. In the context introduced by the previous sections, trimming the aircraft means finding the flight condition and the set of control aerodynamic actions $\Delta C_{F}^{\text {tr }}$, lying inside or on the boundary of the corresponding AMS, so that Eq. (2) is verified.

Trim is cast as a generic constrained optimization problem, for which only the objective function has to be defined on the basis of the desired application study. The following subsections present the various parts of the optimization problem structure, while several implementations of the approach are presented in the following Sect. 6.

\subsection{Assigned trim parameters}

The value of a certain number of variables has to be assigned to assure that the trim problem is well-posed. This is generally requested to prevent the existence of explicit relations among trim controls that are also state variables [1]. For the present formulation, these are collected in Eq. (13). The ground track orientation is a meaningful parameter only in 
Fig. 3 Geometric interpretation of control authority about various motion axes and directions in an $\mathbb{R}^{2}$ Moment Space

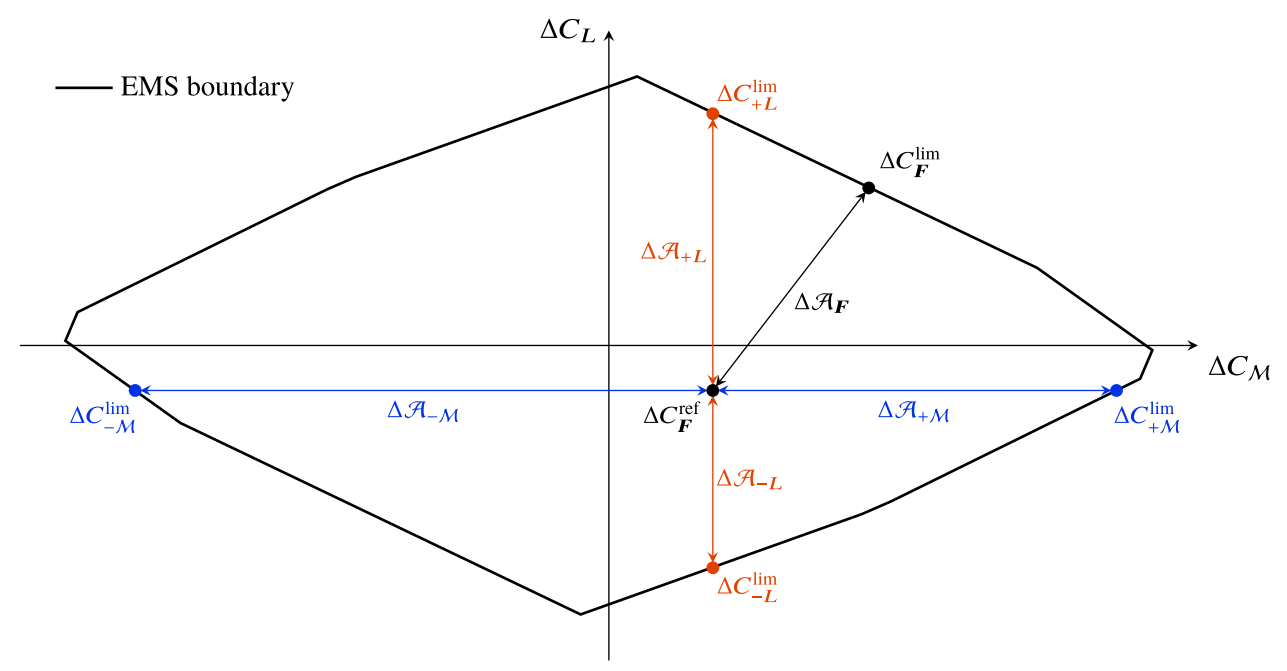

geo-referenced applications, but it has no influence on the outcome of the trim problem.

$\tau_{0}=\left\{X_{E}, Y_{E}, Z_{E}, \psi_{\mathrm{GT}}, V, \dot{\psi}, \dot{\theta}, \dot{\phi}\right\}^{\mathrm{tr}}$

Additional equality constraints may have to be imposed for other trim controls, depending on the application. These can be written in matrix form as in Eq. (14). For example, assigning the value of $\beta$ is not strictly necessary for posing the trim problem correctly, but is desired to uniquely identify the trim flight condition. Similarly, the flight path angle or throttle level may be specified to characterize a particular flight scenario, such as climbing or landing.

$A^{\mathrm{eq}} \boldsymbol{\tau}=b^{\mathrm{eq}}$

\subsection{Trim controls and bounds}

The trim controls set $\tau$ has been conceptually separated into three subsets: the pilot inputs subset $\Pi=\left\{\delta_{\text {lat }}, \delta_{\text {lon }}, \delta_{\text {dir }}, \delta_{T}\right\}$, the aircraft attitudes subset $\boldsymbol{\Theta}=\{\gamma, \psi, \theta, \phi, \beta\}$, and the control forces subset $\Delta C_{F}=\left\{\Delta C_{X}, \Delta C_{Y}, \Delta C_{Z}, \Delta C_{\mathcal{L}}, \Delta C_{\mathcal{M}}, \Delta C_{\mathcal{N}}\right\}$. All pilot inputs are normalized and bounded to the $[-1,1]$ interval, apart from the throttle level which is bounded to $[0,1]$. The attitude angles are bounded to the $[-\pi / 6, \pi / 6]$ interval, while the control force controls are left unbounded.

The $\boldsymbol{I}$ and $\Delta C_{F}$ subsets share the purpose of generating control forces and moments that need to be allocated to the effectors. In these regards, and depending on the architecture of the flight control system, they may have overlapping contributions to the results of the trim problem. Since the current work focuses solely on the application of CA methods, the latter subset is retained for the proposed trim problem formulations, and the effective subset of pilot inputs is reduced to $\boldsymbol{\Pi}=\left\{\delta_{T}\right\}$. The resulting generic set of trim controls used as a baseline for all the presented applications is shown in Eq. (15).

$\tau=\left\{\delta_{T}, \gamma, \psi, \theta, \phi, \beta, \Delta C_{F}\right\}$

\subsection{Linear constraints due to the AMS geometry}

A special set of linear inequality constraints and additional equality constraints is enforced for trim control forces, to imply that each point $\Delta C_{F}$ has to be in the interior or on the boundary of every possible AMS. The linear constraint equations are reported in Eq. (16). The actual value of the constraints comes as the results of the following preliminary optimization problem.

For every relevant direction of Moment Space, flight parameters $\alpha$ and $\beta$, and control effectors positions $\delta$ are varied to obtain the $B$ matrix which results in the AMS spanning the most distance in that direction. Once the most extended AMS in every direction is obtained, the convex hull of all AMSs is calculated. This is not an AMS itself, but rather is the smallest convex set containing all the most extended AMSs. Hence, it serves a good purpose to establish the feasible region for trim control forces.

$A_{\mathrm{CH}}^{\text {ineq }} \Delta C_{\boldsymbol{F}} \leq b_{\mathrm{CH}}^{\text {ineq }}$

$A_{\mathrm{CH}}^{\mathrm{eq}} \Delta C_{\boldsymbol{F}}=b_{\mathrm{CH}}^{\mathrm{eq}}$

The inequalities are always well defined because the convex hull is, by definition, a convex set. The equalities are non-null if any of the edges, facets, or $\left(N_{\boldsymbol{F}}-1\right)$-dimensional elements constituting the boundary of the convex hull is parallel to any reference axis in Moment Space. The $A_{\mathrm{CH}}$ matrices and $b_{\mathrm{CH}}$ column arrays constitute the Linear Constraint 
Representation (LCR) of the convex hull (Eq. 17), and have been calculated using the vert $21 \mathrm{con}$ routine in MATLAB $^{\circledR}[26]$.

$\mathrm{LCR}_{\mathrm{CH}}=\left\{A_{\mathrm{CH}}^{\text {ineq }}, b_{\mathrm{CH}}^{\text {ineq }}, A_{\mathrm{CH}}^{\mathrm{eq}}, b_{\mathrm{CH}}^{\mathrm{eq}}\right\}$

With this approach, the number of inequality constraints depends on the dimension of Moment Space $N_{F}$, and on the number of control effectors $N_{\delta}$. For large problems, it can grow to the order of thousands. An alternative, less strict approach to construct such constraints may consist in wrapping the convex hull in its bounding hyper-rectangle. In this case, the inequality constraints would only be $2 N_{F}$, corresponding to the $2 N_{F}$ boundary elements of the hyperrectangle (e.g. 4 edges of a rectangle in $\mathbb{R}^{2}, 6$ faces of a parallelepiped in $\mathbb{R}^{3}$, etc.).

\subsection{Non-linear constraints}

Most importantly, the non-linear equality constraints of Eq. (18) enforce that the solution of the optimization problem is actually representative of a trim condition, as defined in Eq. (2).

$f\left(\tau_{0}, \tau\right)=0$

Additional inequality constraints may be enforced on the angle of attack, to ensure that the resulting trim conditions are contained in the region described by the aircraft aerodynamic model of Eq. (5). Because $\alpha$ is neither a trim control, nor a state variable, these have to be formulated as nonlinear constraints for the present Flight Mechanics model, but are simply expressed as in Eq. (19).

$\alpha_{\mathrm{lb}} \leq \alpha \leq \alpha_{\mathrm{ub}}$

\subsection{Solution and algorithm}

A flowchart overview of the trim problem formulation and implementation is presented in Fig. 4. At each solver iteration, the control effectiveness matrix $B$ is calculated using the current tentative value of $\alpha$ and $\beta$, and the value of $\delta$ from the previous trim iteration. Hence, the AMS geometry evolves throughout the optimization routine, and is not frozen to the moment of the initialization. This is especially important for the evaluation of control authority. The effectors positions $\boldsymbol{\delta}$ are calculated using the LP-DCA method, as explained in Sect. 4.2.

The optimization problem is solved with the fmincon routine in MATLAB ${ }^{\circledR}$, using the interior-point algorithm. Because the problem is non-smooth, also due to the sharp corners in the geometry of the AMS, the solver stops either by reaching an optimality tolerance of $10^{-3}$, or a step size of $10^{-6}$. All bounds and constraints are respected with a tolerance of $10^{-6}$.

In the next section, several application studies are presented by introducing their objective function, the selected forces in Moment Space, and by analyzing the resulting trim conditions. As it could be expected, the solver finds local optima that, in general, depend on the first-guess values of the trim controls. For this reason, each of the following case studies has been performed twenty times by assigning random initial values to $\Delta C_{F}$, all within the AMS. Only the results with the best value of the respective objective function are reported for brevity.

\section{Applications and results}

For all of the following applications, trim is performed at standard sea level conditions for a horizontal trajectory. The flight path and side-slip angles are specified as additional equality constraints, as shown in Eq. (20).

$\gamma^{\text {tr }}=0$

$\beta^{\text {tr }}=\beta_{0}$

The definitive baseline set of trim controls used in the following applications is then reported in Eq. (21).

$\tau=\left\{\delta_{T}, \psi, \theta, \phi, \Delta C_{\boldsymbol{F}}\right\}$

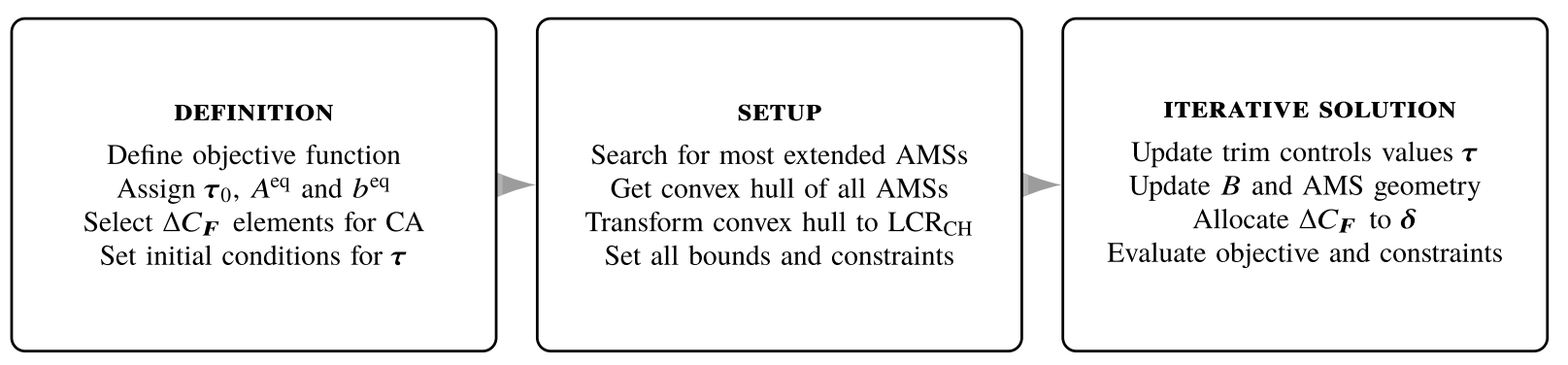

Fig. 4 Flowchart overview of the trim problem formulation and implementation 
The engine throttle is mainly devoted to trimming for the prescribed airspeed. Given a fixed flight path angle, the elevation angle is mainly devoted to trimming for the necessary angle of attack. The remaining attitude angles are devoted to accomplishing the prescribed side-slip and lateral-directional equilibrium. Finally, the allocated control actions are devoted to minor force and moment adjustments, the equilibrium about the aircraft center of mass, and the improvement of the value of the trim objective function.

Section 6.1 presents and discusses both the trim problem for maximum control authority in a given direction and the trim problem for maximum balanced control authority. These approaches must be introduced together to show comprehensive trends in the results, and promote comparisons. Also, since they lay at the core of the most original contribution of the paper, plenty of space is dedicated to them in an attempt to provide the clearest and most complete explanation possible.

Sections 6.2 and 6.3 present and discuss results for the trim problems achieving minimum aerodynamic drag and assigned angle of elevation, respectively. These formulations are extremely well established in the literature (see Sect. 2) or inherently intuitive in nature, and, therefore, do not require any overly detailed introduction.

\subsection{Maximum control authority}

Two approaches are presented for the current application, based on two slightly different definitions of control authority. Both approaches revolve around the search of a limit point for the calculation of the trim objective function. The limit point is calculated by solving a simple optimization sub-problem at every step of the overarching trim problem. The objective functions and their respective limit points are briefly characterized in the following sub-sections before presenting the actual applications and results.

\subsubsection{Definition of control authority about a given axis}

With reference to Eq. (12), trimming the aircraft for maximum control authority about a specified motion axis (i.e. direction of Moment Space) means finding $\Delta C_{F}^{\text {ref }}=\Delta C_{F}^{\text {tr }}$ in the interior or on the boundary of the AMS, so that its distance to $\Delta C_{F}^{\lim }$ is maximum and Eq. (2) is verified.

As already introduced in Sect. 4.2 from a geometric perspective, $\Delta C_{F}^{\text {lim }}$ is the furthest point from $\Delta C_{F}^{\text {ref }}$ which simultaneously belongs to the half-line $\ell_{\boldsymbol{F}}$ and the AMS. Its position results from the solution of Eq. (22), where both the AMS and $\ell_{\boldsymbol{F}}$ can be expressed in terms of the linear equality and inequality constraints corresponding to their respective LCR representations.

$$
\Delta C_{\boldsymbol{F}}^{\mathrm{lim}}=\arg \left\{\begin{array}{cl}
\max _{\Delta C_{F}} & \mathcal{J}^{\text {lim }}=\left\|\Delta C_{\boldsymbol{F}}-\Delta C_{\boldsymbol{F}}^{\mathrm{ref}}\right\|^{2} \\
\text { subj. to } & \Delta C_{\boldsymbol{F}} \in\left\{\text { AMS } \cap \ell_{\boldsymbol{F}}\right\}
\end{array}\right\}
$$

Once the limit point is found, the optimal value of the objective function $\mathcal{J}_{*}^{\lim }$ is the specified control authority for the current reference point $\Delta C_{F}^{\text {ref }}$. This is obviously a function of $\Delta C_{F}^{\text {ref }}$ itself, and to be maximized, the objective function of the overarching trim problem has to be formulated as in Eq. (23).

$$
\max _{\tau} \mathcal{J}^{\mathrm{tr}}=\left\|\Delta C_{F}^{\mathrm{lim}}-\Delta C_{F}\right\|=\mathcal{A}_{F}
$$

\subsubsection{Definition of balanced control authority}

Balanced control authority $\mathcal{A}_{0}$ is here conveniently defined as the average distance to all the vertices of the AMS. Therefore, the point of the AMS with maximum $\mathcal{A}_{0}$ is the point with the minimum sum of distances to all of the AMS vertices. Such limit point $\Delta C_{F}^{0}$ coincides with the centroid of the AMS [27] and, in case all the control effectors position limits are symmetric, with the origin of Moment Space. In general, its position can be calculated by solving the optimization of Eq. (24), where $d_{i}$ is the distance between the candidate point $\Delta C_{F}$ and the $i$ th vertex of the AMS, and $n$ is the number of vertices of the AMS.

$$
\Delta C_{F}^{0}=\arg \left\{\begin{array}{cc}
\min _{\Delta C_{F}} & \mathcal{J}^{0}=\sum_{i=1}^{n} d_{i} \\
\text { subj. to } & \Delta C_{F} \in \text { AMS }
\end{array}\right\}
$$

Once the limit point is found, the overarching trim problem to maximize $\mathcal{A}_{0}$ can be formulated in terms of minimizing the distance between the candidate trim point $\Delta C_{F}^{\text {ref }}$ and $\Delta C_{F}^{0}$ itself, as shown in Eq. (25).

$$
\min _{\tau} \mathcal{J}^{\mathrm{tr}}=\left\|\Delta C_{F}^{0}-\Delta C_{F}\right\|=\mathcal{A}_{0}
$$

In the broader scope of flight dynamics, balanced control authority could assume an important meaning in the assessment of handling qualities characteristics. If the AMS is transformed into an Attainable Acceleration Set, by making use of the aircraft inertia tensor, a flight maneuver achieving maximum balanced control authority would also achieve minimum total acceleration. This could be relevant for handling qualities criteria which define the desired minimum acceleration value corresponding to the best performance, for a specified flight task. 


\subsubsection{Application to symmetric flight with $3 \mathrm{DoF}$}

For this study case, the dynamic simulation is constrained in the vertical plane. The flight condition is longitudinalsymmetric and the aircraft has only $3 \mathrm{DoF}$. Control surfaces are constrained to move symmetrically during the $\mathrm{CA}$ problem, but each left-right pair is free to move according to the LP-DCA algorithm. These simplifications allow to set $\beta_{0}=0$, and to allocate only the control lift and pitch moment: $\Delta C_{F}=\left\{\Delta C_{L}, \Delta C_{\mathcal{M}}\right\}$. It is then possible to visualize the two-dimensional AMS, the trim points and the control authorities in Moment Space, as shown in Fig. 5.

In the figure, several similar AMSs can be distinguished, each corresponding to the maximum control authority about a different direction of Moment Space. This is due to the fact that different control authorities are obtained with different trim values of the angle of attack and control surface deflections. Because aerodynamic control actions are a function of both $\alpha$ and $\delta$, as expressed in the aerodynamic model of Fig. 5, each of the optimum trim points is associated with a slightly different control effectiveness matrix $B$, which results in a slightly different geometry of the AMS. The latter is exploited to calculate the maximum control authority about the prescribed axis.

The position of trim points in the AMS is in line with expectations, and their relative arrangement is preserved for all analyzed airspeed values. A preferential diagonal direction is highlighted by trim points in Moment Space, taking the form of a clear tradeoff between the control lift and pitch moment that are necessary for trim at different angles of attack. For example, a negative control lift is needed to

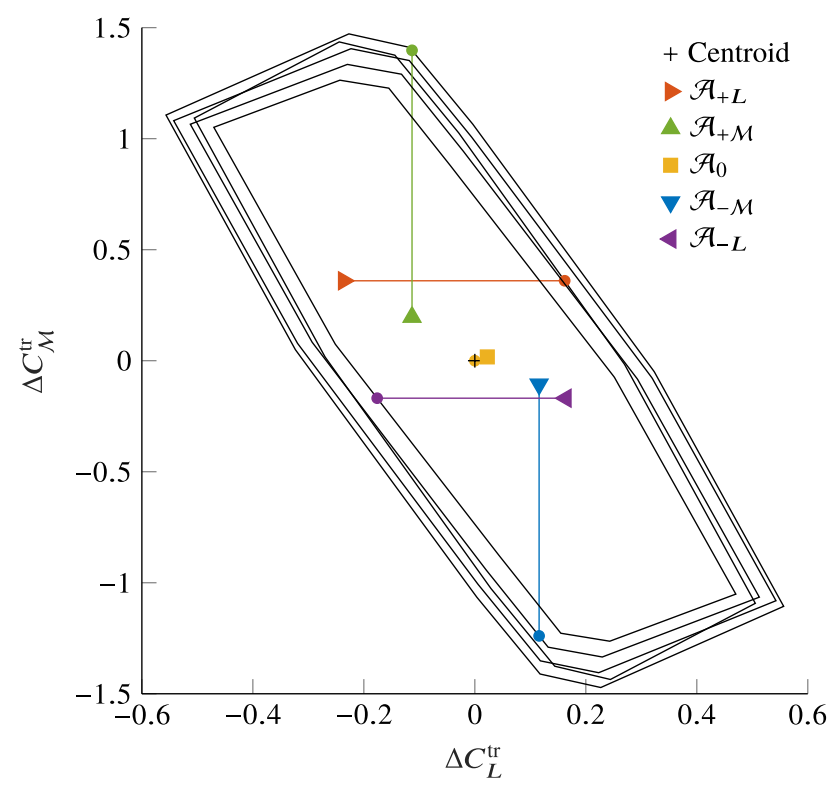

Fig. 5 Position in Moment Space of trim points maximizing control authorities about the pitch and lift axes, for $V^{\mathrm{tr}}=180 \mathrm{~m} / \mathrm{s}$ maximize the lift-up and pitch-up control authority. This corresponds to a positive pitch-up control moment, in a similar way to what happens with conventional aircraft configurations, and a relatively high trim angle of attack. The opposite is true for lift-down and pitch-down control authorities.

The trim point for balanced control authority is very close to the centroid of the AMS, but not precisely coincident with it. This is due to the fact that the aircraft is not trimmable with completely null control surface deflections. For the same reason, the trim points corresponding to the other control authorities are not on the boundary of the AMS. In such conditions, despite maximizing the prescribed objective function, they would not guarantee the compliance with the trim constraints. In all cases, it is evident how the control deflections are deployed to maximize control authority in the given direction, while the angle of attack is adjusted to guarantee vertical equilibrium.

The same study has been repeated at various airspeeds, and the results for control authority and angle of attack are synthetically reported in Fig. 6. Optimum control authority is very different on the basis of the axis it relates to, but it is comparable for the two directions on each axis. For all airspeeds, control power about the pitch axis is greater than the one about the lift axis, while balanced control authorities lies between the two. All angles of attack decrease with increasing airspeed, as it would be expected. The most interesting insights arise when inspecting the relation between the angle of attack and control surface deflections at trim. This is the main focus of the next study case.

\subsubsection{Application to symmetric and asymmetric flight with 6 DoF}

In the present study case, the aircraft is free to move with $6 \mathrm{DoF}$ and all control surfaces are independent, apart from the two rudders which are forced to move as one. The set of allocated forces has been chosen to be the most immediate extension of the classic "three moment" CA problem, namely $\Delta C_{F}=\left\{\Delta C_{L}, \Delta C_{\mathcal{L}}, \Delta C_{\mathcal{M}}, \Delta C_{\mathcal{N}}\right\}$. The lift force has been included in order to be able to maximize control authority about its axis. The symmetric flight condition is investigated by imposing $\beta_{0}=0$, and the asymmetric one by imposing the maximum side-slip required by regulations, $\beta_{0}=V_{\mathrm{SW}} / V^{\mathrm{tr}}[28]$.

As done previously, results are compared for maximum control authority in both directions of the lift and pitch axes, and for maximum balanced control authority. Since the AMS is a subset of $\mathbb{R}^{4}$ for this application, results cannot be shown in Moment Space. Attention is focused on the optimal control surfaces deflections at trim, shown in Fig. 7, and on the other trim controls, reported in Table 2. Control surface deflections on the main wings are positive if with trailing edge down, negative if with trailing edge up. The vertical 


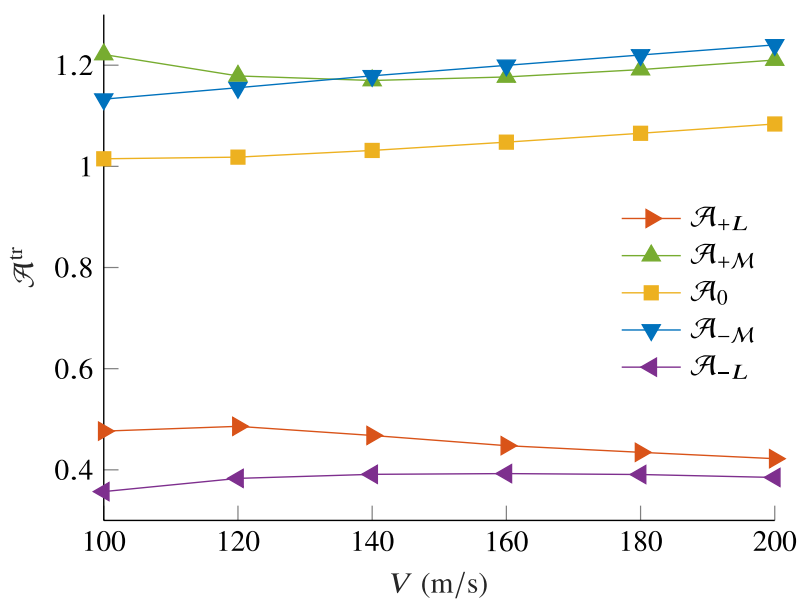

(a) Control authority

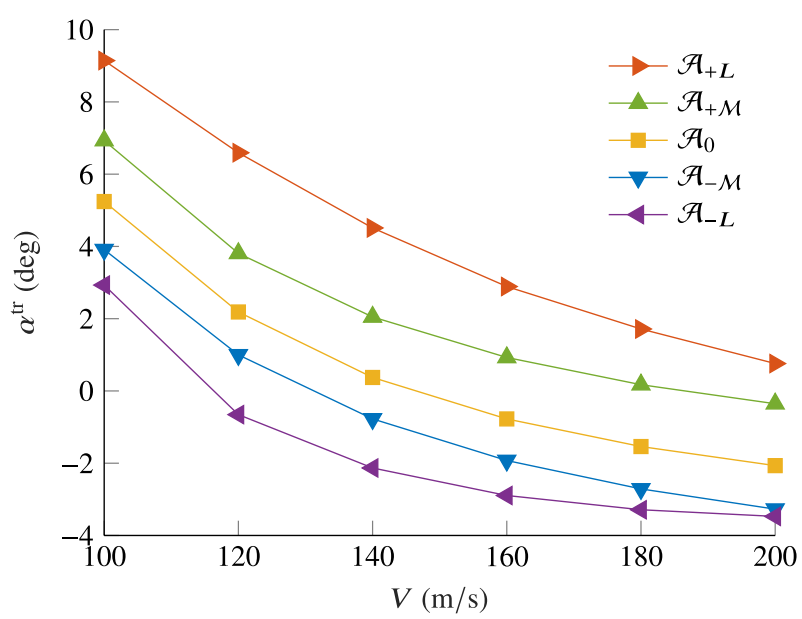

(b) Angle of attack

Fig. 6 Maximum control authority and corresponding angle of attack for various airspeeds and directions in Moment Space

axis in the figure has been inverted to reflect this convention. Rudder deflections are positive if they cause the aircraft nose to point left of the flight path.

For the symmetric flight condition, control surface deflections on the front wing range from the positive to the negative saturation limit, depending on which control authority is maximized. Deflections are practically symmetric, although not perfectly so. This is most likely due to numerical precision, and explains the very small, non-null value of the bank angle. Maximum lift-up control authority is achieved with very negative deflections, both on the front and on the rear wing, paired up with a significant positive angle of attack $\alpha^{\mathrm{tr}}=5.1^{\circ}$. Maximum lift-down control authority is achieved with mostly large positive deflections at a negative angle of attack of $\alpha^{\text {tr }}=-4.5^{\circ}$. This reflects the behavior seen in the previous study case, and clearly shows how the optimizer trades $\alpha$-generated lift with $\delta$-generated lift in order to maximize the specified control authority.

In a similar way, the maximum pitch-down control authority is achieved by exploiting the pitch-down moment due to the propulsion system. By increasing the throttle level at a very low angle of attack, the solver finds a trim condition with drastic positive deflections on the front wing and negative deflections on the rear wing. Such coupling between the horizontal and rotational equilibrium is not observed for $\mathcal{A}_{+L}$, where the slightly higher throttle setting can be justified by the higher magnitude of the corresponding angle of attack.

Trim conditions for maximum pitch-up control authority and maximum balanced control authority are overall very similar. Both cases are characterized by the same throttle setting, and by small deflections on both the front and the rear wing. As just observed, pitch control moment can be manipulated more significantly than control lift through the alteration of thrust. But to maximize pitch-up control authority with a propulsive system placed above the aircraft center of gravity, thrust should be reduced as much as possible. To achieve horizontal equilibrium with low thrust, drag must also be kept low by employing small deflections of the control surfaces. The same reasoning in inverse order can be carried out to explain the results for the $\mathcal{A}_{0}$ case.

For the asymmetric flight condition, all control surface deflections show a similar behavior, slightly stretching in magnitude according to which control authority is maximized. The front inner surfaces are adjusted asymmetrically to provide for the necessary roll moment, together with the two tail rudders correcting for the necessary yaw moment. The front outer surfaces and all the rear ones are then adjusted for optimizing control authority. Trends in the remaining flight parameters are overall less evident and the numerical values less extreme. This happens because more control power is required to achieve basic trim, leaving less available control authority to the objective function.

Control surfaces on the front wing show more complex behavior than the ones on the rear wing, which are deflected by the same angle in all cases. This can be justified by the fact that front control surfaces have a smaller moment arm with respect to the aircraft center of gravity. Hence, they are able to alter control lift with a small impact on control pitch, and they may be preferred by the solver as they do not cause important coupling effects.

\subsection{Minimum aerodynamic drag}

In each of the previous applications, a given CA method has been used to explore the properties of trim conditions 


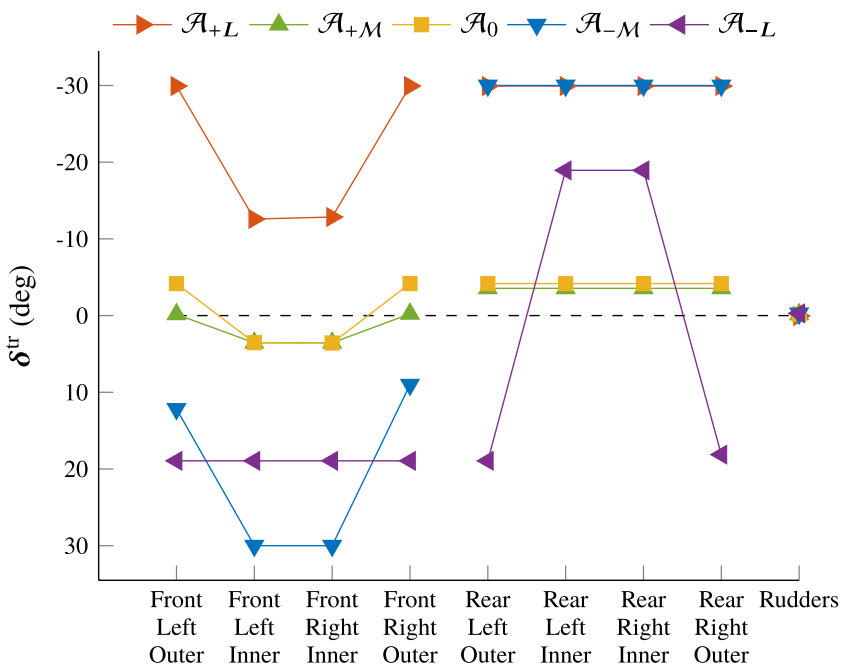

(a) Symmetric flight, $\beta_{0}=0^{\circ}$

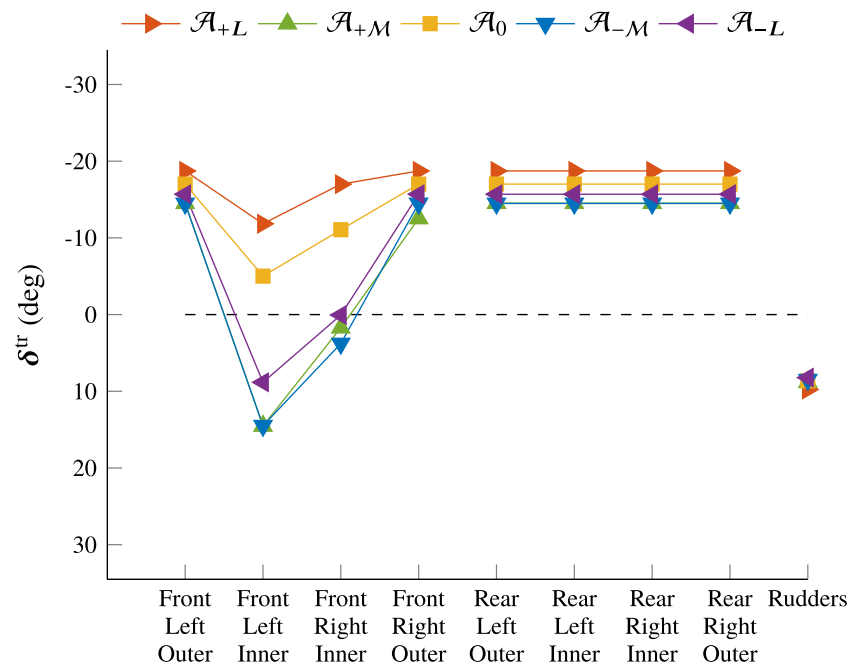

(b) Asymmetric flight, $\beta_{0}=4.3^{\circ}$.

Fig. 7 Control surface deflections maximizing control authorities about different directions in Moment Space, for $V^{\mathrm{tr}}=170 \mathrm{~m} / \mathrm{s}$

Table 2 Trim controls for symmetric and asymmetric trimmed flight, maximizing control authority about different directions in Moment Space, for $V^{\mathrm{tr}}=170 \mathrm{~m} / \mathrm{s}$

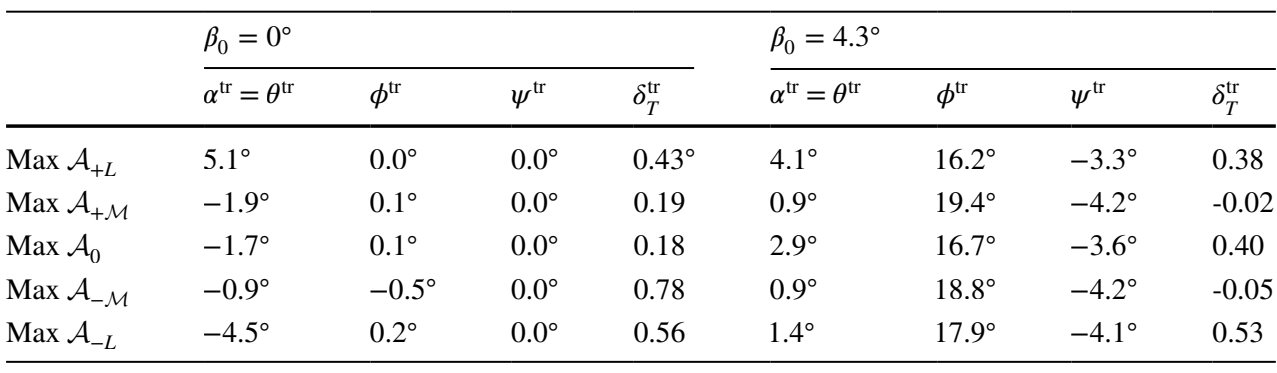

maximizing control authority in different directions of Moment Space. In the present section, a given, more traditional, objective function is optimized using different AMS dimensions and a conventional ganging strategy for control surfaces. The intention is to study the impact of the AMS geometry on the optimal objective function value. The present application aims at minimizing total aerodynamic drag at trim conditions, as shown in Eq. (26). The dynamic simulation is constrained to $3 \mathrm{DoF}$ longitudinal-symmetric flight, with $\beta_{0}=0$.

$\min _{\tau} \mathcal{J}^{\mathrm{tr}}=C_{D}$

In the first two study cases, the trim problem formulation presented in Sect. 5 has been implemented. The first study case employs the two-dimensional CA approach already seen in Sect. 6.1.3, consisting in the allocation of only the control lift and pitch moment: $\Delta C_{F}=\left\{\Delta C_{L}, \Delta C_{\mathcal{M}}\right\}$. This is synthetically referred, in the following figures, as the "AMS 2D" case. The second study case explores a threedimensional CA problem, where control drag is additionally included in the trim controls set, hence setting $\Delta C_{F}=\left\{\Delta C_{D}, \Delta C_{L}, \Delta C_{\mathcal{M}}\right\}$. This is referred to as the "AMS
3D" case. As done previously, control surfaces are only constrained to move symmetrically during the CA problem, but each left-right pair is free to move according to the LP-DCA algorithm.

In the third and last study case, trim is performed by using a conventional mechanical flight control system, which links pilot inputs to the control effectors by means of a constant gearing and ganging matrix. Therefore, only in this case, the control forces subset $\Delta C_{F}$ is completely replaced by the pilot inputs subset $\boldsymbol{\Pi}$, introduced in Sect. 5.2. All control surfaces are constrained to move symmetrically, the inboard ones move in opposition (as conventional elevators), while the outboard ones move in agreement (as DLC effectors).

The resulting minimum trim drag has been reported in Fig. 8 for various airspeeds. In all cases, regardless of the CA method and the AMS dimensionality, the aircraft motion is constrained in the vertical plane and retains $3 \mathrm{DoF}$. All drag coefficient curves with respect to airspeed follow the expected $C_{D} \propto V^{-4}$ relation. The numerical values of the drag coefficient may appear slightly lower than one could expect for this aircraft category. This is due to the fact that 


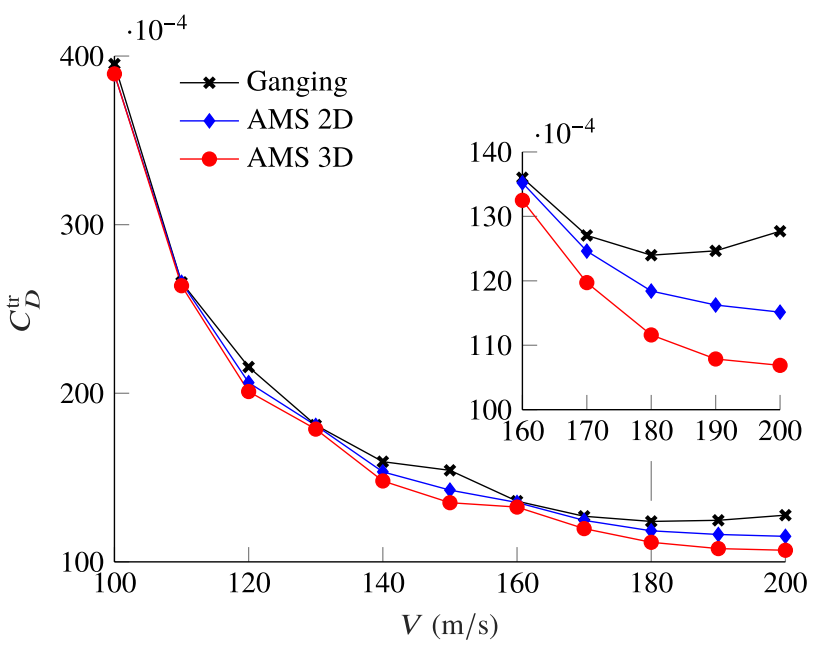

Fig. 8 Minimum trim drag as a function of airspeed using different CA methods. The enlargement shows that different CA methods achieve different minimum trim drag at different airspeeds, with the AMS 3D approach performing the best

the underlying aerodynamic model has been generated with the VSAERO panel method code [17], which is not capable to capture parasitic and compressible drag effects very accurately, as already mentioned in Sect. 3. Nevertheless, because the aircraft geometry and the aerodynamic model are exactly the same for all three CA formulations presented in this section, the comparison among drag curves is deemed fair.

The AMS 3D formulation is able to achieve a slightly lower minimum drag coefficient at any airspeed. This is probably due to the inclusion of information on $\Delta C_{D}$ in the geometry of the AMS itself. Conventional ganging gives the worst performance overall, as expected. For $V=200 \mathrm{~m} / \mathrm{s}$, the corresponding drag curve has already passed its minimum, while the curve of the AMS 3D case has not flattened yet. At this airspeed, the difference between drag coefficients at trim is maximum and equal to about 20 drag counts, as it can be appreciated in the enlargement reported in the same Fig. 8.

Figure 9 shows the corresponding trim angle of attack and throttle. All CA methods perform very similarly, but a trend is visible especially at higher values of the airspeed. The angle of attack and throttle levels are also comparable for the three approaches. The conventional ganging method converges to a smaller $\alpha^{\text {tr }}$ if compared to the ones based on the AMS geometry. On the other hand, it requires a slightly higher throttle level for every airspeed. Interestingly, both the angle of attack and the throttle curves for the minimum drag solution are similar in shape and values, although not equal, to the solutions for the maximum balanced control authority study presented in Sect. 6.1, which have not been reported for brevity.

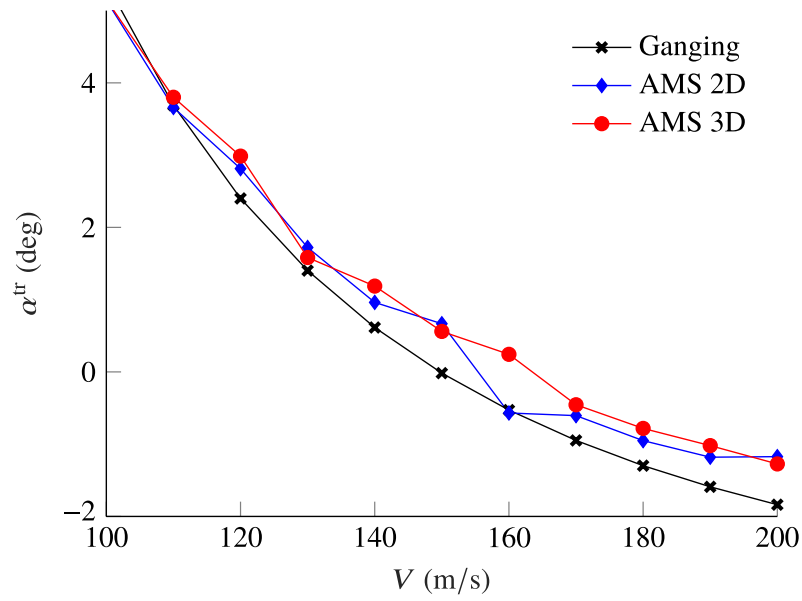

(a) Angle of attack

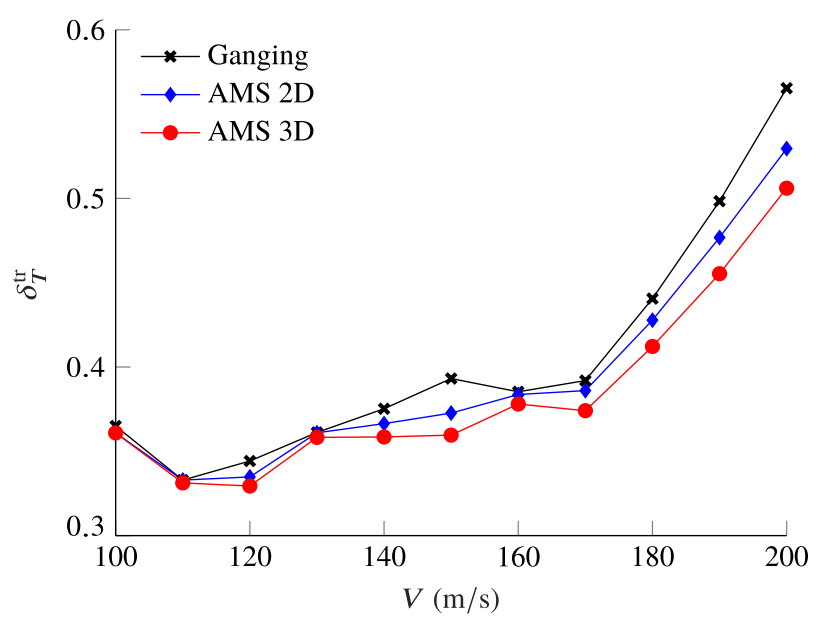

(b) Throttle level

Fig. 9 Trim angle of attack and throttle level as a function of airspeed, using different CA methods for the minimum drag condition

\subsection{Assigned angle of elevation}

For this last application, the objective function to be minimized is the absolute difference between the achieved and a prescribed value of the angle of elevation at trim. All the assumptions from the previous section hold, with the difference that only the two-dimensional CA problem, allocating lift and pitch moment, has been implemented in this case. This is in light of the small differences among the results of the previous study, and due to drag being irrelevant to the analysis of the present flight scenario. Therefore, the trim control forces subset is $\Delta C_{F}=\left\{\Delta C_{L}, \Delta C_{\mathcal{M}}\right\}$ and the objective function is reported in Eq. (27).

$\min _{\tau} \mathcal{J}^{\mathrm{tr}}=\left\|\theta-\theta^{\mathrm{ref}}\right\|$ 
Such an objective function has been chosen to test the box-wing capabilities to trim the aircraft at precise body attitudes, and to obtain the limits within which this task is possible for a given aircraft model. This can be desired for flight tasks which require precise attitude hold, such as air refueling, for example.

Results are shown in Fig. 10, and correlate the achieved value of the trim elevation angle to an array of prescribed values for the same angle. The horizontal segments of each curve highlight the minimum and maximum elevation angle at which trim is achievable for the given airspeed. The diagonal segments between these values delineate the region for which it is possible to trim the aircraft for any specified elevation angle at the given airspeed. In almost all cases, the extreme values of $\theta^{\text {tr }}$, which is also equal to $\alpha^{\text {tr }}$ because $\gamma_{0}=0$, coincide with the values of the angle of attack that maximize the control authorities shown in Fig. 6b. This confirms, once again, that the solver presented in Sect. 6.1 is able to push $\alpha$-generated lift to its limit to optimize $\delta$-generated lift and pitch moment.

\section{Conclusions}

A generic trim problem formulation has been presented in the form of a constrained optimization problem, which employs forces and moments due to the aircraft control effectors as trim controls. A LP DCA method has been used to map the control forces and moments to the corresponding control effectors positions. The geometry of the AMS is used in all cases for defining a set of linear equality and inequality

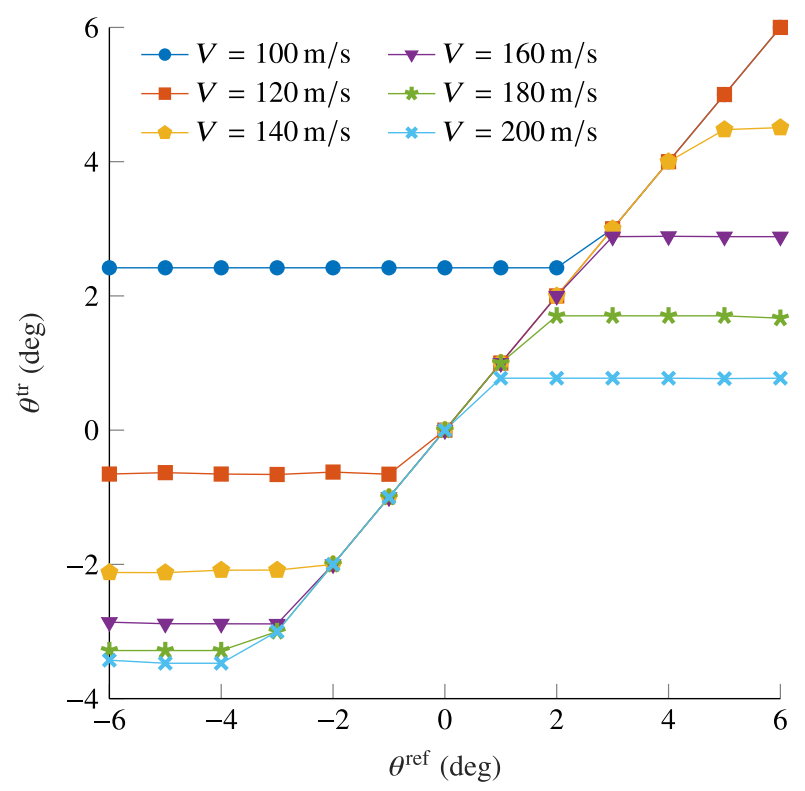

Fig. 10 Achieved and prescribed trim elevation angles as a function of airspeed, using the AMS 2D CA method constraints, which ensure that control forces and moments are attainable by the control effectors.

A definition of control authority has been given, which is entirely based on control forces and moments, independent of the aircraft configuration and/or number of control effectors. It has been interpreted geometrically as a distance between a reference point and a limit point within the AMS.

With application to an innovative box-wing aircraft called PrandtlPlane, the trim methodology is used to compare trim conditions for maximum control authority in the pitch axis, in the lift axis, and for maximum balanced control authority about all motion axes. For these applications, the AMS geometry is also used at each solver iteration to calculate the value of the objective function. Results show that the method is able to capitalize on the angle of attack or the throttle setting to obtain the control surfaces deflections which maximize control authority in the assigned direction.

Another application has been performed to minimize total aerodynamic drag, using a 2D or 3D AMS geometry. The former involves the allocation of control lift and pitch moment, while the latter also includes control drag. These approaches have also been compared to a standard trim problem formulation, which relies on the pilot stick input rather than control forces as trim controls, and does not employ any Control Allocation method. The three formulations all perform similarly under every point of view, but the one using the 3D AMS achieves slightly smaller drag, probably thanks to the inclusion of control drag in the AMS geometry. Trim conditions for this application are overall similar to those achieved for maximum balanced control authority.

Lastly, an application study to achieve an assigned value of the elevation angle has been carried out. This flight scenario was deemed interesting to investigate the ability of box-wing aircraft to (partially) decouple lift and pitch moment control. The extreme values of trim elevation angle achieved coincide with the values of the angle of attack that maximize control authorities in the first study case. This confirms, once again, that the solver is able to push $\alpha$-generated lift to its limit in order to optimize $\delta$-generated lift and pitch moment.

The optimization problem is iterative and non-smooth in nature, and therefore only capable of finding local optima. These usually depend on the initial condition chosen for the selected trim controls. Therefore, several optimization runs of the same flight condition may be required to find a global optimum. Because of this, the proposed method can only be used off-line, for the generation of trim databases to be accessed at later stages, in faster applications.

Future research efforts can be devoted to improving the presented methodology when strongly non-linear aerodynamics is involved, or when searching for optimum trim conditions in the drag rise airspeed range. For example, the trim formulation has proven able to successfully minimize 
total aerodynamic drag, but optimization of control authority about the drag axis is still problematic due to chattering. In particular, in proximity of the combination of control surface deflections resulting in minimum control drag, all the elements in the drag row of the control effectiveness matrix tend to zero. This leads the AMS to collapse about one dimension in Moment Space (e.g. from 3D to 2D) and deteriorates the numerical calculation of the linear constraints representing its geometry, as well as the calculation of control authority as the objective function. Including control drag as an allocated force could be interesting to explore flight scenarios like trim in steep descent conditions.

Lastly, time domain simulations are recommended to study the impact of the selected control authority on maneuvering flight starting from the achieved trim condition. Given a specific maneuver to be performed after trim is achieved, a criterion to find which control authority has to be optimized to obtain best maneuver performance could be sought.

Funding The research presented in this paper has been carried out in the framework of the PARSIFAL (Prandtlplane ARchitecture for the Sustainable Improvement of Future AirpLanes) research project, which has been funded by the European Union within the Horizon 2020 Research and Innovation Program (Grant agreement n.723149).

\section{Declarations}

Conflict of interest The authors declare that they have no conflict of interest.

Open Access This article is licensed under a Creative Commons Attribution 4.0 International License, which permits use, sharing, adaptation, distribution and reproduction in any medium or format, as long as you give appropriate credit to the original author(s) and the source, provide a link to the Creative Commons licence, and indicate if changes were made. The images or other third party material in this article are included in the article's Creative Commons licence, unless indicated otherwise in a credit line to the material. If material is not included in the article's Creative Commons licence and your intended use is not permitted by statutory regulation or exceeds the permitted use, you will need to obtain permission directly from the copyright holder. To view a copy of this licence, visit http://creativecommons.org/licenses/by/4.0/.

\section{References}

1. Stevens, B.L., Lewis, F.L., Johnson, E.N.: Aircraft Control and Simulation. Wiley, New York (2016)

2. Durham, W., Bordignon, K.A., Beck, R.: Aircraft Control Allocation. Wiley, New York (2017)

3. Bodson, M.: Evaluation of optimization methods for control allocation. J. Guid. Control Dyn. 25(4), 703-711 (2002). https://doi. org/10.2514/2.4937

4. Varriale, C., Voskuijl, M., Veldhuis, L.L.: Trim for maximum control authority using the attainable moment set. In: AIAA Scitech 2020 Forum. American Institute of Aeronautics and Astronautics (2020). https://doi.org/10.2514/6.2020-1265
5. Voskuijl, M., de Klerk, J., van Ginneken, D.: Flight mechanics modeling of the PrandtlPlane for conceptual and preliminary design. In: Springer Optimization and Its Applications, pp. 435-462. Springer US (2012). https://doi.org/10.1007/ 978-1-4614-2435-219

6. de Wringer, S., Varriale, C., Oliviero, F.: A generalized approach to operational, globally optimal aircraft mission performance evaluation, with application to direct lift control. Aerospace 7(9), 134 (2020). https://doi.org/10.3390/aerospace 7090134

7. De Marco, A., Duke, E., Berndt, J.S.: A general solution to the aircraft trim problem. In: AIAA Modeling and Simulation Technologies Conference and Exhibit (2007). https://doi.org/10.2514/6. 2007-6703

8. Goodrich, K.H., Sliwa, S.M., Lallman, F.J.: A closed-form trim solution yielding minimum trim drag for airplanes with multiple longitudinal-control effectors. Technical Publication NASATP-2907. https://ntrs.nasa.gov/citations/19890014097 (1989). Accessed 11 Nov 2021

9. Garmendia, D.C., Mavris, D.N.: Alternative trim analysis formulations for vehicles with redundant multi-axis control surfaces. J. Aircr. 53(1), 60-72 (2016). https://doi.org/10.2514/1.C033184

10. Beck, R.E.: Application of Control Allocation Methods to Linear Systems with Four or More Objectives. PhD Thesis, Virginia Tech. http://hdl.handle.net/10919/28088 (2002). Accessed 11 Nov 2021

11. Durham, W.C., Bolling, J.G., Bordignon, K.A.: Minimum drag control allocation. J. Guid. Control Dyn. 20(1), 190-193 (1997). https://doi. org/10.2514/2.4018

12. Stolk, R., de Visser, C.: Minimum drag control allocation for the innovative control effector aircraft. In: 5th CEAS Conference on Guidance, Navigation and Control (2019)

13. Cipolla, V., Abu Salem, K., Picchi Scardaoni, M., Frediani, A., Binante, V.: Preliminary design and performance analysis of a boxwing transport aircraft. In: AIAA SciTech Forum, Orlando, Florida, USA (2020). https://doi.org/10.2514/6.2020-0267

14. Carini, M., Meheut, M., Kanellopoulos, S.: Aerodynamic analysis and optimization of a boxwing architecture for commercial airplanes. In: AIAA SciTech Forum, Orlando, Florida, USA (2020). https://doi.org/ 10.2514/6.2020-1285

15. Prandtl, L.: Induced drag of multiplanes. NACA Technical Note 182, National Advisory Committee for Aeronautics (1924)

16. Raju Kulkarni, A., Varriale, C., Voskuijl, M., La Rocca, G., Veldhuis, L. L.: Assessment of sub-scale designs for scaled flight testing. In: AIAA Aviation: Forum. American Institute of Aeronautics and Astronautics, June 2019 (2019). https://doi.org/10.2514/6.2019-3089

17. Nathman, J.K.: VSAERO 7.9: A Computer Program for Calculating the Nonlinear Aerodynamic Characteristics of Arbitrary Configurations. Stark Aerospace, Inc., Redmond (2016)

18. van Ginneken, D., Voskuijl, M., van Tooren, M., Frediani, A.: Automated control surface design and sizing for the prandtl plane. In: 51st AIAA/ASME/ASCE/AHS/ASC Structures, Structural Dynamics, and Materials Conference. American Institute of Aeronautics and Astronautics (2010). https://doi.org/10.2514/6.2010-3060

19. Varriale, C., Raju Kulkarni, A., La Rocca, G., Voskuijl, M.: A hybryd, configuration-agnostic approach to aircraft control surface sizing. In: 25th International Congress of the Italian Association of Aeronautics and Astronautics (AIDAA) (2019a)

20. Voskuijl, M., La Rocca, G., Dircken, F.: Controllability of blended wing body aircraft. In 26th Congress of International Council of the Aeronautical Sciences (2008)

21. Varriale, C., Hameeteman, K., Voskuijl, M., Veldhuis, L.L.: A thrust-elevator interaction criterion for aircraft optimal longitudinal control. In: AIAA Aviation 2019 Forum. American Institute of Aeronautics and Astronautics (2019b). https://doi.org/10.2514/6. 2019-3001 
22. Bolender, M.A., Doman, D.B.: Method for determination of nonlinear attainable moment sets. J. Guid. Control Dyn. 27(5), 907-914 (2004). https://doi.org/10.2514/1.9548

23. Bordignon, K.A.: Constrained control allocation for systems with redundant control effectors. PhD Thesis, Virginia Tech. http://hdl. handle.net/10919/28570 (1996). Accessed 11 Nov 2021

24. Johansen, T.A., Fossen, T.I.: Control allocation-a survey. Automatica 49(5), 1087-1103 (2013). https://doi.org/10.1016/j.autom atica.2013.01.035

25. Frost, S.A., Bodson, M., Burken, J.J., Jutte, C.V., Taylor, B.R., Trinh, K.V.: Flight control with optimal control allocation incorporating structural load feedback. J. Aerosp. Inf. Syst. 12(12), 825-834 (2015). https://doi.org/10.2514/1.I010278

26. Jacobson, M.: Analyze n-dimensional polyhedra in terms of vertices or (in)equalities. https://bit.ly/2VwEKbq (2017). Accessed 11 Nov 2021
27. Carbó-Dorca, R.: A study on the centroid vector of a polyhedron. J. Math. Chem. 54(1), 61-71 (2015). https://doi.org/10.1007/ s10910-015-0548-9

28. European Aviation Safety Agency. Certification Specifications for Large Aeroplanes CS-25A19. EASA (2017)

29. Varriale C, Voskuijl M (2021) A Control Allocation approach to induce the center of pressure position and shape the aircraft transient response. Aerosp Sci Technol 119:107092. https://doi. org/10.1016/j.ast.2021.107092

Publisher's Note Springer Nature remains neutral with regard to jurisdictional claims in published maps and institutional affiliations. 